Japan, and, I may add, the West Indies, it would appear that the crust of the globe is practically in a constant state of tremor. The variations in these movements are more law-abiding than the large earth movements, and they show a direct relationship to barometric fluctuation.

Their relationship to many other telluric and atmospheric phenomena, together with their cause, has yet to be discovered. As every one has the opportunity to observe these phenomena, they call for attention. Just as a turbulent sea outraces a coming typhoon and gives mariners warning of approaching danger, it is possible that these microscopic disturbances of the soil may hold connection with subsequent phenomena, and lead us by their study to the better understanding of the complexity of phenomena with which we are surrounded.

Tokio, Japan

JOHN MILNE

\section{THE MECHANICAL THEORY OF MAGNETISM}

J $F$ Prof. Hughes were as great a master of writing English as he is of experimenting, his views on magnetism would receive speedier acceptation, for they would then probably be understood without that close study which his involved sentences and heterogeneous paragraphs now demand. It is very remarkable that such an ardent worker, such a deep thinker, and such a clear and simple experimenter should have such difficulty in expounding his views on paper. His experimental demonstrations are always clear and convincing, his recent lecture at the Royal Institution appealed to every degree of intelligence present, but his papers at the Royal Society want some strong external directing influence to render their meaning evident.

What is magnetism, according to this expert philosopher? It is an inherent quality of the molecules of matter, as determined and constant as that of their gravity, affinity, or cohesion, and like these qualities it differs in degree with every kind of matter. He does not attempt at present to define it closer than this. We cannot tel what gravity is, neither need we say what magnetism is. All Prof. Hughes says is that every molecule in nature is a little magnet imbued with a certain polarity varying in degree but constant for each substance, in virtue of which it has a north and a south pole along the same axis, and that the only change that takes place is a change in the direction of this polar axis. When these molecules are symmetrically arranged by some external directing influence, so that all their poles lie in the same direction, we have evident magnetism. Iron becomes a magnet in virtue of the fact that its molecules are free to move under the influence of external magnetic action, while copper is not a magnet because its molecules are immovable and irresponsive to the same cause. Steel becomes permanently magnetised because its molecules are rigid, and retain the axial direction impressed upon them. Soft iron is readily demagnetised because its molecules have great freedom of motion. Coercive force is therefore simply absence of freedom of molecular motion-it is, indeed, molecular rigidity. The extent to which the axis of polarity can be deflected from its normal direction is its point of saturation.

Evident magnetism is the symmetrical arrangement of the polarised molecules along one line; neutrality is symmetrical arrangement of the same molecules in closed curves. In both cases the sum of the magnetic influence of all the molecules is the same; but in evident magnetism it is directed outwards, in neutrality it is directed inwards. Remaining magnetism is partial neutrality. The experimental way in which Prof. Hughes demonstrated these conclusions is the most beautiful investigation he has yet made. He proves the existence of the same polarity in the atmosphere and in the ether, and he attributes diamagnetic effects to the higher magnetic capacity of the ether than of the substances suspended in it. It is therefore a differen- tial action. Molecules, moreover, have inertia-they resist being put in motion; and when in motion they resist stoppage-they possess momentum. The direction of the axis of polarity can be displaced by the physical fcrces, such as mechanical stress, heat, or electricity. He shows that mechanical motion, heat, and electricity are of similar kind-they are vibratory, or some mode of motion. Magnetism, however, he considers not to be a mode of motion, and therefore it is not a physical force. It is simply an arrangement of the molecules of matter in symmetry or dissymmetry under the influence of some physical force. He seems to imply, though he does not directly say so, that the influence of electric currents upon magnets is not due to any direct action between them, but to the fact that the currents have polarised the ether in which both are suspended.

His views are very broad and highly suggestive, but there are some points that are not clear and that demand further elucidation. Why, for instance, does mechanical elongation and contraction take place when bars of iron are magnetised and demagnetised ? How can heat and strong sonorous vibrations be produced unless there be a considerable expenditure of energy? How does he account for the attractive and repulsive properties of magnets, and for magnetic induction? He has certainly wrested magnetism from the realms of hypothesis and brought it within the domain of theory. The days of Coulomb and Poisson's fluids and Ampère's elementary currents of electricity are over; the molecular character of magnetism is experimentally established; but what is a molecule, and how becomes it polarised unless it be in rotation? How does the external directing influence act? We are also inclined to ask, Has Prof. Hughes sufficiently grasped Ampère's theory? It was purely mathematical, based on the assumption of the circulation of currents around each molecule. He goes no further than Ampere did, for he has not answered the question, What is polarity? In fact his polarised molecules are all little magnets, and no theory of magnetism will be complete until it explains these little magnets. Thus the difference between Ampere and Hughes is the difference between a current and a magnet.

However, on the assumption that a molecule is a magnet, Prof. Hughes has built up a very complete theory, which he has demonstrated experimentally in a way that places him in the very front rank of experimental philosophers.

\section{NOTES}

THE number of candidates up this session for the Fellowship of the Royal Society is sixty-seven.

WE understand that Sir Joseph Hooker has been nominated one of the vice-presidents for the Montreal meeting of the British Association. Instead of Mr. Crookes, Prof. W. G. Adams will give one of the public lectures. For the reduction of the fares of members the sum of 14,000 dollars has been allotted, only tho e elected at or before the Southampton meeting being entitled to share in the subsidy. This is in addition to the liberal reductions that will be made by the steamship and railway companies. All the American railways will reduce their fares by one-haif. The American Association, which meets at Philadelpbia on September 3, has given a cordial invitation to the Montreal vis tors to take part in its meetings and excursions. Those wishing to share in the subsidy of 14,000 dollars must apply before September 25. For the Aberdeen meeting in 1885 , Sir I.yon Playfair will be proposed as president. A well-attended meeting of the Organising Committee of the Chemical Section has been held under the presidency of Prof. Roscoe. Promises of papers were received from several well-known chemists, and a small executive committee was formed to draw up a list of papers and to communicate with Canadian and American 